\title{
Changes in cannabis use according to socioeconomic status among Finnish adolescents from 2000 to 2015
}

\author{
Noora Knaappila ${ }^{1 *}$, Mauri Marttunen$^{2}$, Sari Fröjd ${ }^{1}$, Nina Lindberg ${ }^{3}$ and Riittakerttu Kaltiala1,4,5
}

\begin{abstract}
Background: Despite reduced sanctions and more permissive attitudes toward cannabis use in the USA and Europe, the prevalences of adolescent cannabis use have remained rather stable in the twenty-first century. However, whether trends in adolescent cannabis use differ between socioeconomic groups is not known. The aim of this study was to examine trends in cannabis use according to socioeconomic status among Finnish adolescents from 2000 to 2015.
\end{abstract}

Methods: A population-based school survey was conducted biennially among 14-16-year-old Finns between 2000 and 2015 ( $n=761,278)$. Distributions for any and frequent cannabis use over time according to socioeconomic adversities were calculated using crosstabs and chi-square test. Associations between any and frequent cannabis use, time, and socioeconomic adversities were studied using binomial logistic regression results shown by odds ratios with 95\% confidence intervals.

Results: At the overall level, the prevalences of lifetime and frequent cannabis use varied only slightly between 2000 and 2015. Cannabis use was associated with socioeconomic adversities (parental unemployment in the past year, low parental education, and not living with both parents). The differences in any and frequent cannabis use between socioeconomic groups increased significantly over the study period.

Conclusions: Although the overall changes in the prevalence of adolescent cannabis use were modest, cannabis use increased markedly among adolescents with the most socioeconomic adversities. Socioeconomic adversities should be considered in the prevention of adolescent cannabis use.

Keywords: Adolescent, Cannabis, Epidemiology, Socioeconomic factors, Socioeconomic status, Parental factors, Finland

\section{Background}

In Finland, about 17\% reported having used cannabis at some point in their lives (Varjonen 2012). The prevalence of cannabis use increased from the 1990s to the 2000s in the overall population, although it seems to have leveled off in the 2010s (Karjalainen et al. 2016; Varjonen 2012). Similarly, the attitudes toward cannabis use have become more permissive among Finns (Karjalainen et al. 2016). According to the ESPAD study

\footnotetext{
* Correspondence: noora.knaappila@tuni.fi

${ }^{1}$ Faculty of Medicine and Life Sciences, University of Tampere, 33014

Tampere, Finland

Full list of author information is available at the end of the article
}

conducted in 2015, eight percent of Finnish adolescents had tried cannabis in their lifetime (ESPAD Group 2016). As with cannabis use among adults, increase in adolescent cannabis use was rapid from the 1990s to the mid-2000s but slowed down after that (ESPAD Group 2016). The Finnish cannabis policy rests on total prohibition, which denotes that both use, possession, manufacturing, and selling are considered illegal (EMCDDA 2017), and the policy has not fundamentally changed in recent years (Varjonen 2012).

Despite being decriminalized in many countries, cannabis use has several detrimental effects on health that cannot be disregarded. The most comprehensive 
evidence exists on the association between cannabis use and mental health disorders, such as depression, anxiety, suicidal ideation, substance use, and psychosis among people with other genetic or environmental vulnerabilities (Chadwick et al. 2013; Karila et al. 2014; MacDonald and Pappas 2016; Patel et al. 2017; Volkow et al. 2014). Cannabis use also increases the risk for respiratory diseases and traffic accidents (Karila et al. 2014; MacDonald and Pappas 2016; Patel et al. 2017; Volkow et al. 2014). Furthermore, adolescent cannabis use is associated with social marginalization (Babor et al. 1978; Dugré et al. 2017; Varjonen 2012). In addition to individual suffering, drug-related harms cause substantial public expenditures, the largest parts in Finland going toward the enforcement of public order and safety, court and prison costs, and social services (Varjonen 2012).

Risk factors of cannabis use include easy availability (von Sydow et al. 2002), male gender (Gfroerer et al. 2007; ter Bogt et al. 2014; Thompson 2001), other substance use (Hayatbakhsh et al. 2009), peer and parental influences (Melchior et al. 2011; von Sydow et al. 2002), and positive attitudes toward cannabis (von Sydow et al. 2002). In addition, the association between cannabis use and socioeconomic status (SES) has been studied in the scientific literature. SES depicts an individual's or group's relative position within a society (Galobardes et al. 2006a; Galobardes et al. 2006b). It can be measured at both individual, household, and neighborhood levels (Krieger et al. 1997). It can be assessed not only through individual measures, such as education, income, or occupation (Galobardes et al. 2006a) but also through composite measures that provide an overall index of socioeconomic level. The measurement of adolescent SES is challenging as the final education and income levels are yet to be accomplished in adolescence. Therefore, parental SES is often used as a proxy measure of adolescent SES (Torikka et al. 2014; Torikka et al. 2017; Knaappila et al. 2018; Knaappila et al. 2019a; Knaappila et al. 2019b). Low socioeconomic status is a wellestablished risk factor of morbidity and mortality in all age groups (Clegg et al. 2009; Lund Jensen et al. 2017; Rawshani et al. 2016; Saydah et al. 2013). It affects health through several mechanisms, including living circumstances, lifestyle, and the consumption of health services (Øvrum 2011; Stringhini et al. 2011; Wang et al. 2015).

Of the socioeconomic factors, adolescent cannabis use has been associated with low parental education and not living with both parents (Delva et al. 2005; Henkel and Zemlin 2016; Swift et al. 2008; Widome et al. 2013). However, studies on the association between cannabis use and socioeconomic status are scarce. Substances tend to be more easily available in less affluent neighborhoods (Karriker-Jaffe 2013). According to Willis (1977), adolescents with low socioeconomic status may perceive certain behaviors that are common in low-SES groups as a reinforcement to their identities. Therefore, adolescents from low socioeconomic backgrounds may view substance use, that is more common in low-SES groups than in other socioeconomic groups, as something to pursue in an effort to reinforce their identity.

Scientific evidence suggests that recent decades have seen an increase in socioeconomic health disparities in the Nordic countries, including Finland. Matthiessen et al. (2014) observed that the prevalence of overweight increased in Denmark between 2000 and 2008 in boys of parents with low educational level only. Similarly, socioeconomic disparities increased in self-rated health among Swedish adolescents between 2002 and 2014 (Ahlborg et al. 2017). Torikka et al. $(2017,2014)$ found that the differences in the prevalences of depression, frequent drinking, and drunkenness between socioeconomic groups increased among Finnish adolescents from 2000 to 2011. Therefore, although changes in the general prevalence of cannabis use have been modest in the twenty-first century, there may be differing trends between socioeconomic groups. However, studies investigating socioeconomic trends in adolescent cannabis use have not yet been conducted.

\section{Methods}

The aim of this study was to examine time-trends in the prevalence of cannabis use according to socioeconomic status among Finnish adolescents. Data for the study was obtained from The School Health Promotion Study of the National Institute for Health and Welfare, which is a survey on the health, health behavior, and school experiences of Finnish adolescents. The survey has been conducted biennially since 1996 among Finnish 8th and 9th graders with pooled 2-year data. In this study, we used data collected between 2000 and 2015. The data were collected anonymously during a school lesson under the supervision of a teacher, who did not interfere with the responses. Participants were informed orally and in writing about the voluntary nature of the study, and returning the survey was considered consent to participate. The survey was done by paper and pencil and it took about $30-45 \mathrm{~min}$ to complete. After this, the surveys were put in an envelope, sealed, and returned directly to the research center. The timing of the study, sampling, and data collection methods were held constant in each survey. Altogether, 761,278 (50,404-109, 127 biennially) 8th and 9th graders participated in the survey. 8th graders were 14-15 years old and 9th graders 15-16 years old at the time of the surveys. The biennial cohorts covered between 74 and $84 \%$ of the whole age cohort of the country. The study was approved by the ethics committee of Pirkanmaa Hospital District and the National Institute of Health and Welfare. 
Lifetime substance use was elicited as follows: "Have you ever tried or used the following substances? Please answer every item." The response alternative measuring lifetime cannabis use was "marijuana or hashish." The formulation of the question was held constant across all questionnaires, except that in the 2015 questionnaire the response alternative was slightly more specified: "marijuana or hashish (cannabis)." The response alternatives were "never/once/2-4 times/5 times or more often." For the analyses, two dichotomous variables"any cannabis use" (at least once vs never) and "frequent cannabis use" (5 times or more often vs other)-were created. The cut-off point of five or more times was chosen as Zoccolillo et al. (1999) demonstrated that such frequency indicates problematic use in this age group.

The socioeconomic variables recorded were parental education, parental unemployment in the past year, and family structure. The associations between all three variables and adolescent health are well established (Areba et al. 2018; Sleskova et al. 2006; Torikka et al. 2017). Parental education was elicited as follows: "What is the highest educational qualification your father/mother has achieved?" The response options in the 2000 questionnaire were "basic school/vocational school/high school and/or vocational school/university or polytechnic." The response options varied a little over time: for instance, in the 2013 questionnaire there was a response option "no education," which was removed again in the 2015 questionnaire. For the analyses, parental education was dichotomized as parental basic education only (including the response alternatives "basic school" and "no education") versus other. Parental unemployment was elicited as follows: "Have your parents been unemployed or laid off work during the past YEAR?" The response alternatives were the same in all questionnaires: "neither/one parent/both parents." The family structure was elicited as follows: "My family consists of..." The response options in the 2000 questionnaire were "mother and father/mother and stepfather/father and stepmother/mother only/father only/spouse/other caregiver." The response options varied slightly over time. For the analyses, family structure was dichotomized as living with both parents versus other. In this article, all three variables are referred to as socioeconomic adversities. In addition, a variable "cumulative socioeconomic adversity" was created, in which all three socioeconomic variables were combined: a score of 0 stood for having no socioeconomic adversities (living with both parents, no parental unemployment, at least one parent with higher than basic education) and a score of 4 stood for having all socioeconomic adversities (not living with both parents, both parents unemployed, both parents with basic education only). The prevalences of socioeconomic adversities over time are presented elsewhere (Knaappila et al. 2018).
The distributions of any and frequent cannabis use across years among boys and girls in the whole sample as well as according to cumulative socioeconomic adversity were studied using crosstabulation and chi-square test. Less than $3 \%$ of the responses were missing in all variables apart from parental education, which had missing values of $7.6 \%$ in boys and $6.6 \%$ in girls. As all the results were statistically significant $(p<0.01)$, the $p$ values were not addressed in the tables. Bivariate associations were studied using binomial logistic regression results shown by odds ratios with $95 \%$ confidence intervals. Any and frequent cannabis use were entered as dependent variables. In the first model, categorical time periods (2000-2001, 2002-2003, 2004-2005, 2006-2007, 20082009, 2010-2011, 2012-2013, 2014-2015) were entered as an independent factor using the time period 20002001 as a reference category. In the second model, family structure (living with both parents/other), parental unemployment in the past year (neither/one parent/ both parents), and parental education (both parents basic education only/other) were entered as an independent factor one at a time. In the third model, the file was split according to categorical time periods and cumulative socioeconomic adversity was entered as an independent factor.

\section{Results}

Of girls $6 \%$ and of boys $8 \%$ reported having used cannabis at least once in their lifetime. Of girls $2 \%$ and of boys $3 \%$ reported having used cannabis five times or more often (Table 1). Both any and frequent cannabis use varied only slightly between years among both sexes (Table 2).

The associations between cannabis use and socioeconomic adversities are presented in Table 3. Any and frequent cannabis use were more common among girls and boys who did not live with both parents than among those who lived with both parents. Cannabis use was positively associated with parental unemployment: any and frequent cannabis use were most common among girls and boys whose both parents had been unemployed and least common among those whose parents had not been unemployed in the past year. Any and frequent cannabis use were also more common when both parents had only basic education than when at least one parent had higher than basic education. Socioeconomic differences were greater in frequent cannabis use than any cannabis use.

The more socioeconomic adversities accumulated, the more common any and frequent cannabis use were. Prevalences in any and frequent cannabis use increased markedly among adolescents with most cumulative socioeconomic adversity over time (Table 4). Differences in any cannabis use between girls not living with both 
Table 1 Distribution of any and frequent cannabis use and socioeconomic adversities among boys and girls in the 8th or 9th grades of comprehensive school in Finland between 2000 and $2015(\%)$

\begin{tabular}{|c|c|c|}
\hline & Boys $(n=381527)$ & Girls $(n=376814)$ \\
\hline Age, years (mean $(s d))$ & $15.4(0.7)$ & $15.3(0.6)$ \\
\hline \multicolumn{3}{|l|}{ 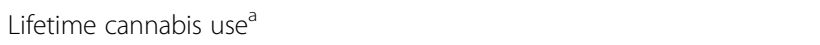 } \\
\hline Yes & 8.1 & 6.0 \\
\hline No & 90.7 & 93.3 \\
\hline \multicolumn{3}{|l|}{ Frequent cannabis use ${ }^{\mathrm{b}}$} \\
\hline Yes & 2.9 & 1.5 \\
\hline No & 95.9 & 97.9 \\
\hline \multicolumn{3}{|l|}{ Lives with both parents } \\
\hline Yes & 74.4 & 73.7 \\
\hline No & 23.3 & 25.1 \\
\hline \multicolumn{3}{|c|}{ Both parents only basic education } \\
\hline Yes & 5.6 & 5.9 \\
\hline No & 86.8 & 87.5 \\
\hline \multicolumn{3}{|c|}{ Parental unemployment past year } \\
\hline No & 70.9 & 69.9 \\
\hline One parent & 23.6 & 25.6 \\
\hline Both parents & 3.2 & 3.3 \\
\hline
\end{tabular}

ased cannabis at least once in one's lifetime

bUsed cannabis at least five times in one's lifetime

parents, with both parents unemployed and parental basic education only and girls living with both parents, with no parental unemployment, and at least one parent with higher than basic education increased from 2000$2001(\mathrm{OR}=4.6,95 \% \mathrm{CI} 2.8-7.7)$ to $2014-2015(\mathrm{OR}=$ 36.6, 95\% CI 23.9-55.9). Similarly, differences in any cannabis use between socioeconomic groups increased among boys from 2000-2001 (OR $=12.0$, 95\% CI 8.2$17.6)$ to $2014-2015(\mathrm{OR}=51.4,95 \%$ 36.6-72.0). Socioeconomic differences in frequent cannabis use among girls increased from 2000-2001 (OR $=7.9$, 95\% CI 3.617.4 ) to 2014-2015 (OR = 101.9, 95\% CI 64.1-162.3). Likewise, socioeconomic differences in frequent cannabis use increased among boys from 2000-2001 (OR = 29.5, 95\% CI 19.7-44.2) to 2014-2015 (OR = 101.9, 95\% CI 64.1-162.3) (Table 5).

\section{Discussion}

In this study, we found that cannabis use was associated with socioeconomic adversities among Finnish adolescents. The prevalences of any and frequent cannabis use were higher among adolescents not living with two parents than among those living with both parents. Similarly, the prevalences of any and frequent cannabis use were higher among adolescents with parental unemployment in the past year and adolescents with parental basic education only than among adolescents without these socioeconomic adversities. The more socioeconomic adversities an adolescent had, the more likely they used cannabis. The most important, and novel, finding was that cannabis use increased markedly from 2000 to 2015 among adolescents with most socioeconomic adversities.

The association between cannabis use and low parental education, as well as that between adolescent cannabis use and not living with both parents, have been observed in earlier studies (Delva et al. 2005; Henkel and Zemlin 2016; Swift et al. 2008; Widome et al. 2013). To our knowledge, no previous studies have investigated the association between adolescent cannabis use and parental unemployment. There are many possible explanations for these associations. First, all the socioeconomic adversities studied are associated with lower income level in the family, which in turn is a risk factor of adolescent cannabis use ( $\mathrm{Tu}$ et al. 2008; von Sydow et al. 2002). Parents with higher education level may have more knowledge on the harms of cannabis and therefore more negative attitudes toward cannabis use (Wu et al.

Table 2 Distribution of any ${ }^{a}$ and frequent ${ }^{b}$ cannabis use across years among boys and girls in the 8th or 9th grades of comprehensive school in Finland (\%)

\begin{tabular}{|c|c|c|c|c|c|c|c|c|}
\hline \multicolumn{9}{|l|}{ Boys } \\
\hline & $\begin{array}{l}2000-2001 \\
(n=42685)\end{array}$ & $\begin{array}{l}2002-2003 \\
(n=51309)\end{array}$ & $\begin{array}{l}2004-2005 \\
(n=53499)\end{array}$ & $\begin{array}{l}2006-2007 \\
(n=54841)\end{array}$ & $\begin{array}{l}2008-2009 \\
(n=54433)\end{array}$ & $\begin{array}{l}2010-2011 \\
(n=51329)\end{array}$ & $\begin{array}{l}2012-2013 \\
(n=50223)\end{array}$ & $\begin{array}{l}2014-2015 \\
(n=25147)\end{array}$ \\
\hline Any cannabis use & 9.1 & 9.1 & 7.1 & 6.1 & 6.5 & 8.6 & 9.8 & 10.1 \\
\hline Frequent cannabis use & 2.9 & 3.0 & 2.4 & 2.1 & 2.2 & 3.2 & 3.7 & 5.0 \\
\hline \multicolumn{9}{|l|}{ Girls } \\
\hline & $\begin{array}{l}2000-2001 \\
(n=42122)\end{array}$ & $\begin{array}{l}2002-2003 \\
(n=49481)\end{array}$ & $\begin{array}{l}2004-2005 \\
(n=51979)\end{array}$ & $\begin{array}{l}2006-2007 \\
(n=54286)\end{array}$ & $\begin{array}{l}2008-2009 \\
(n=54216)\end{array}$ & $\begin{array}{l}2010-2011 \\
(n=51216)\end{array}$ & $\begin{array}{l}2012-2013 \\
(n=49255)\end{array}$ & $\begin{array}{l}2014-2015 \\
(n=25257)\end{array}$ \\
\hline Any cannabis use & 8.0 & 7.7 & 6.0 & 4.6 & 4.4 & 5.8 & 6.4 & 6.2 \\
\hline Frequent cannabis use & 1.8 & 1.7 & 1.3 & 1.0 & 1.0 & 1.3 & 1.7 & 2.2 \\
\hline
\end{tabular}

aUsed cannabis at least once in one's lifetime

bused cannabis at least five times in one's lifetime 
Table 3 Odds ratios (95\% confidence intervals) for any ${ }^{a}$ and frequent ${ }^{b}$ cannabis use according to socioeconomic adversities among Finnish boys and girls in the 8th and 9th grades of comprehensive school in 2000-2015

\begin{tabular}{|c|c|c|c|c|}
\hline & \multicolumn{2}{|l|}{ Boys } & \multicolumn{2}{|l|}{ Girls } \\
\hline & Any cannabis use & Frequent cannabis use & Any cannabis use & Frequent cannnabis use \\
\hline \multicolumn{5}{|l|}{ Family structure } \\
\hline Living with both parents & Ref & Ref & Ref & Ref \\
\hline Not living with both parents & $2.7(2.6-2.7)$ & $3.1(3.0-3.3)$ & $2.8(2.7-2.8)$ & $3.7(3.5-3.9)$ \\
\hline \multicolumn{5}{|l|}{ Both parents with low education } \\
\hline No & Ref & Ref & Ref & Ref \\
\hline Yes & $1.9(1.8-2.0)$ & $3.2(3.1-3.4)$ & $1.5(1.4-1.5)$ & $2.6(2.4-2.8)$ \\
\hline \multicolumn{5}{|l|}{ Parental unemployment } \\
\hline Neither parent & Ref & Ref & Ref & Ref \\
\hline One parent & $1.5(1.4-1.5)$ & $1.4(1.3-1.5)$ & $1.6(1.5-1.6)$ & $1.7(1.6-1.8)$ \\
\hline Both parents & $4.6(4.4-4.8)$ & $8.4(7.9-8.8)$ & $3.1(2.9-3.2)$ & $6.1(5.6-6.6)$ \\
\hline
\end{tabular}

a Used cannabis at least once in one's lifetime

bused cannabis at least five times in one's lifetime

2015). On the other hand, adults with low education are more likely to use substances (Weitoft et al. 2008), and parental substance use is a major risk factor of adolescent substance use (Hoffmann and Cerbone 2002). The prevalence of mental health problems, which are associated with cannabis use, is also higher among children not living with both parents and those with parental unemployment (Kedzior and Laeber 2014; Pedersen et al. 2001). The associations were stronger for frequent cannabis use than lifetime cannabis use, which is in accordance with the knowledge that socioeconomic adversities are more strongly associated with heavier substance use (Lund 2015; Torikka et al. 2017).

Changes in the prevalence of both lifetime and frequent cannabis use were modest in the overall population. This finding is in accordance with earlier studies (Arnarsson et al. 2018; Delva et al. 2005; Gfroerer et al. 2007; Henkel and Zemlin 2016; Johnson et al. 2015; ESPAD Group 2016; ter Bogt et al. 2014). However, among adolescents with most socioeconomic adversities, the likelihood of both lifetime and frequent cannabis use increased significantly over the study period. The finding is surprising as time trends in adolescent cannabis use according to the socioeconomic status have not been studied previously. One explanation to the findings might be liberalized attitudes toward cannabis use in Finland (Karjalainen et al. 2016). Wardle and Steptoe (2003) observed in their study that unhealthy attitudes were associated with low socioeconomic status. Attitudinal changes may have affected the increase in cannabis use among adolescents with most socioeconomic adversities. In addition, increased disparities in substance use among adults might explain the widening disparities in cannabis use among adolescents (Rotko et al. 2011). Also, availability of cannabis has increased in Finland due to anonymous online markets (Nurmi et al. 2017), which may have increased cannabis use among those most prone to use it. The increasing engagement of youth in the online world may have contributed differently to the behaviors of adolescents in different socioeconomic groups.

Cannabis use is strongly correlated with smoking, and recently increased socioeconomic disparities have also been observed in adolescent smoking (Knaappila et al. 2019a). Increased disparities in smoking may therefore have enhanced disparities in cannabis use. Increased socioeconomic health disparities have also been observed in other areas of adolescent health in Finland and other countries (Knaappila et al. 2018; Knaappila et al. 2019b; Matthiessen et al. 2014; Moraeus et al. 2014; Torikka et al. 2014; Torikka et al. 2017). Torikka et al. (2017, 2014) observed increased socioeconomic differences in depression and alcohol consumption among Finnish adolescents between 2000 and 2011. Similarly, Knaappila et al. $(2018,2019 b)$ observed that the prevalences of delinquency and bullying at school increased among adolescents with low socioeconomic status only. In Finland, the economic depression in the 1990s led to marked increases in unemployment (Rotko et al. 2011). Also, the purchasing power of people on social security benefits has decreased in the past decades (Rotko et al. 2011). These societal changes may have contributed to increases in socioeconomic health disparities in Finland. It can also be that the identity processes of adolescents from different socioeconomic backgrounds are differing in a way that has led to increased socioeconomic disparities in health behaviors among adolescents (Willis 1977). As the socioeconomic disparities are rooted in societal structures, socioeconomic health disparities can be decreased through socio-political decision-making. 
Table 4 Distributions of any ${ }^{\mathrm{a}}$ and frequent ${ }^{\mathrm{b}}$ cannabis use according to socioeconomic adversities ${ }^{\mathrm{c}}$ across years among boys and girls in the 8th or 9th grades of comprehensive school in Finland (\%)

\begin{tabular}{|c|c|c|c|c|c|c|c|c|}
\hline & $2000-2001$ & 2002-2003 & 2004-2005 & 2006-2007 & 2008-2009 & 2010-2011 & 2012-2013 & 201 \\
\hline \multicolumn{9}{|c|}{ Boys, any cannabis use } \\
\hline \multicolumn{9}{|c|}{ Number of socioeconomic adversities } \\
\hline 0 & 6.8 & 6.5 & 4.9 & 3.9 & 4.5 & 5.9 & 6.2 & 5.7 \\
\hline 1 & 9.5 & 10.1 & 8.0 & 6.7 & 7.2 & 9.2 & 10.2 & 10.2 \\
\hline 2 & 15.4 & 16.7 & 12.0 & 12.3 & 11.6 & 15.1 & 15.9 & 15 \\
\hline 3 & 20.8 & 24.9 & 24.0 & 25.5 & 27.1 & 26.2 & 26.7 & 27.3 \\
\hline 4 & 46.8 & 58.9 & 58.5 & 65.6 & 71.6 & 71.4 & 61.1 & \\
\hline
\end{tabular}

Girls, any cannabis use

Number of socioeconomic adversities

$\begin{array}{llll}0 & 5.9 & 5.6 & 3.9 \\ 1 & 8.3 & 8.5 & 6.9 \\ 2 & 12.7 & 12.8 & 10.9 \\ 3 & 14.3 & 17.3 & 15.1 \\ 4 & 22.4 & 32.6 & 44.9\end{array}$

$3.9-29$

6.9

10.9

15.1

44.9

Boys, frequent cannabis use

Number of socioeconomic adversities

$\begin{array}{lll}0 & 1.9 & 1.9 \\ 1 & 2.7 & 3.0 \\ 2 & 5.2 & 5.9 \\ 3 & 10.6 & 12.5 \\ 4 & 35.8 & 44.2\end{array}$

Girls, frequent cannabis use

Number of socioeconomic adversities

\begin{tabular}{llllllllll}
0 & 1.1 & 1.0 & 0.6 & 0.5 & 0.6 & 0.6 & 0.7 & 0.7 \\
1 & 2.0 & 1.7 & 1.5 & 1.2 & 1.0 & 1.3 & 1.6 & 2.2 \\
2 & 3.2 & 3.2 & 2.4 & 2.4 & 1.8 & 2.4 & 3.3 & 4.2 \\
3 & 4.0 & 6.1 & 5.3 & 5.9 & 5.4 & 4.9 & 5.6 & 7.5 \\
4 & 8.2 & 16.3 & 33.7 & 27.8 & 38.2 & 36.4 & 31.0 & 43.5 \\
\hline
\end{tabular}

a Used cannabis at least once in one's lifetime

bUsed cannabis at least five times in one's lifetime

'Socioeconomic adversities included not living with both parents, both parents with basic education only, and both parents unemployed

Securing everyone's equal access to education, work, and social and health services are important ways to decrease socioeconomic disparities in health and well-being, including cannabis use. In addition, socioeconomic adversities should be considered in the prevention of cannabis use among adolescents.

This study has some limitations. Self-report data is susceptible to errors, such as recall bias and invalid responding. Parental education especially may be difficult for an adolescent to recall, which may have caused the proportion of missing responses to be higher on that question than other questions. However, the proportions of missing responses on all questions studied were small and fluctuated slightly without displaying any clear trend that could influence the trends of interest. Invalid responding is another source of error in studies relying on self-report data. Social desirability may result in too low reporting of problem behaviors (Fisher and Katz 2008), and adolescents may also find it funny to exaggerate their symptoms and problem behaviors in questionnaires (Robinson-Cimpian 2014). Such influences on cannabis use were not controlled for, but there is no reason to assume that either social desirability or exaggerating problems biased the trends identified.

Despite the limitations, this study has several strengths: it is based on a unique nationwide time trend data with a large sample size consisting of Finnish 8th and 9th graders $(n=761,278)$. The biennial cohorts covered between 74 and $84 \%$ of the whole age cohort of the country. The sampling and timing of the study as well as 


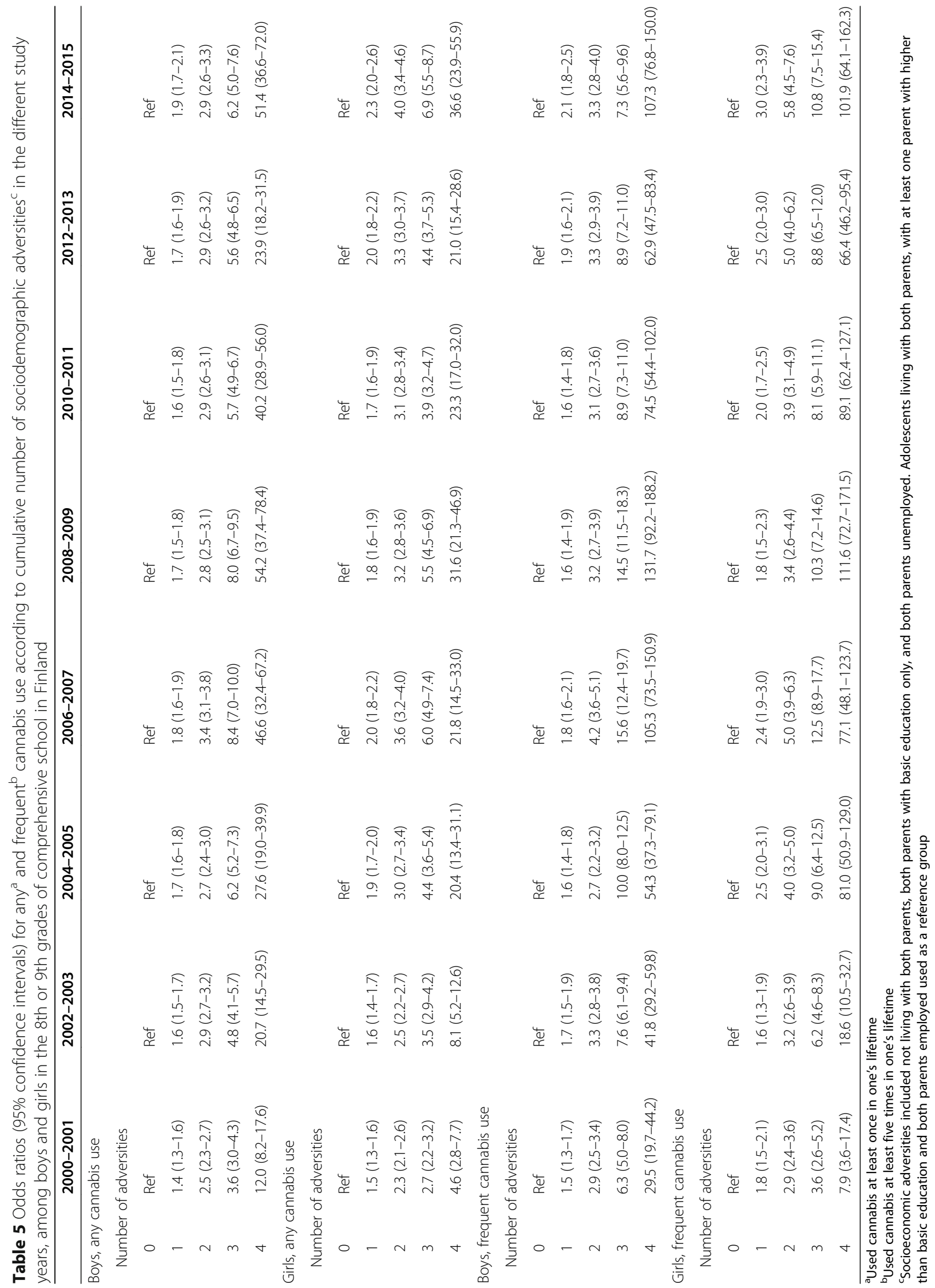


the formulation of questions were held constant over the years. Instead of one measure of cannabis use, we were able to study both any and frequent use. In addition, several measures were used to assess socioeconomic adversity.

\section{Conclusion}

This study found that socioeconomic disparities in cannabis use increased among Finnish adolescents between 2000 and 2015. Although changes in the overall prevalence of cannabis use were modest, cannabis use increased among adolescents with most socioeconomic adversities. Socioeconomic health disparities increase individual suffering and inflict a great burden on public health and economy (Koskinen and Martelin 2007). Securing everyone's equal access to education, work, and social and health services are important ways to decrease socioeconomic disparities in health and well-being, including cannabis use. In addition, socioeconomic adversities should be considered in the prevention of cannabis use among adolescents.

\section{Authors' contributions}

NK carried out the statistical analyses, produced the draft of the manuscript and corrected the final manuscript. The author(s) read and approved the final manuscript.

\section{Competing interests}

The authors declare that they have no competing interests.

\section{Author details}

${ }^{1}$ Faculty of Medicine and Life Sciences, University of Tampere, 33014 Tampere, Finland. ${ }^{2}$ Adolescent Psychiatry, University of Helsinki and Helsinki University Hospital, PO Box 22, Fl-00014 Helsinki, Finland. ${ }^{3}$ Department of Adolescent Psychiatry, Helsinki University Central Hospital, PO Box 590, 00029 HUS, Helsinki, Finland. "Vanha Vaasa Hospital, Vierinkiventie 1, 65380 Vaasa, Finland. ${ }^{5}$ Department of Adolescent Psychiatry, Tampere University Hospital, 33380 Pitkäniemi, Finland.

Received: 2 March 2020 Accepted: 13 November 2020

Published online: 24 December 2020

\section{References}

Ahlborg M, Svedberg P, Nyholm M, Morgan A, Nygren JM. Socioeconomic inequalities in health among Swedish adolescents - adding the subjective perspective. BMC Public Health. 2017;17:838 https://doi.org/10.1186/s12889017-4863-x

Areba EM, Eisenberg ME, McMorris BJ. Relationships between family structure, adolescent health status and substance use: does ethnicity matter? J Community Psychol. 2018:46(1):44-57 https://doi.org/10.1002/jcop.21915.

Arnarsson A, Kristofersson GK, Bjarnason T. Adolescent alcohol and cannabis use in Iceland 1995-2015. Drug Alcohol Rev. 2018;37:S49-57 https://doi.org/10. $1111 /$ dar. 12587

Babor TF, Mendelson JH, Uhly B, Kuehnle JC. Social effects of marijuana use in a recreational setting. Int J Addict. 1978;13:947-59.

Chadwick B, Miller ML, Hurd YL. Cannabis use during adolescent development: susceptibility to psychiatric illness. Front Psychiatry. 2013;4:129 https://doi. org/10.3389/fpsyt.2013.00129.

Clegg LX, Reichman ME, Miller BA, Hankey BF, Singh GK, Lin YD, Goodman MT, Lynch CF, Schwartz SM, Chen WW, Bernstein L, Gomez SL, Graff JJ, Lin CC, Johnson NJ, Edwards BK. Impact of socioeconomic status on cancer incidence and stage at diagnosis: selected findings from the surveillance, epidemiology, and end results: National Longitudinal Mortality Study. Cancer Causes Control. 2009;20:417-35 https://doi.org/10.1007/s10552-008-9256-0.
Delva J, Wallace JM, O'Malley PM, Bachman JG, Johnston LD, Schulenberg JE, Schulenberg JE. The epidemiology of alcohol, marijuana, and cocaine use among Mexican American, Puerto Rican, Cuban American, and other Latin American eighth-grade students in the United States: 1991-2002. Am J Public Health. 2005;95:696-702 https://doi.org/10.2105/AJPH.2003.037051.

Dugré JR, Dellazizzo L, Giguère C-É, Potvin S, Dumais A. Persistency of cannabis use predicts violence following acute psychiatric discharge. Front Psychiatry. 2017:8:176 https://doi.org/10.3389/fpsyt.2017.00176.

EMCDDA, 2017. Cannabis legislation in Europe: an overview. Luxembourg. https://doi.org/10.2810/4682.

ESPAD Group. ESPAD Report 2015: results from the European School Survey Project on alcohol and other drugs. Luxembourg: Publications Office of the European Union; 2016.

Fisher R, Katz J. Social-desirability bias and the validity of self-reported values. Psychol Mark. 2008;17:105-20.

Galobardes B, Shaw M, Lawlor DA, Lynch JW, Davey Smith G. Indicators of socioeconomic position (part 1). J Epidemiol Community Health. 2006a;60(1): 7-12 https://doi.org/10.1136/jech.2004.023531.

Galobardes B, Shaw M, Lawlor DA, Lynch JW, Davey Smith G. Indicators of socioeconomic position (part 2). J Epidemiol Community Health. 2006b;60: 95-101 https://doi.org/10.1136/jech.2004.028092.

Gfroerer JC, Larson SL, Colliver JD. Drug use patterns and trends in rural communities. J Rural Health. 2007;23:10-5 https://doi.org/10.1111/j.17480361.2007.00118.x.

Hayatbakhsh MR, Najman JM, Bor W, O'Callaghan MJ, Williams GM. Multiple risk factor model predicting cannabis use and use disorders: a longitudinal study. Am J Drug Alcohol Abuse. 2009;35:399-407 https://doi.org/10.3109/ 00952990903353415.

Henkel D, Zemlin U. Social inequality and substance use and problematic gambling among adolescents and young adults: a review of epidemiological surveys in Germany. Curr Drug Abuse Rev. 2016;9:26-48.

Hoffmann JP, Cerbone FG. Parental substance use disorder and the risk of adolescent drug abuse: an event history analysis. Drug Alcohol Depend. 2002;66:255-64.

Johnson RM, Fairman B, Gilreath T, Xuan Z, Rothman EF, Parnham T, Furr-Holden CDM. Past 15-year trends in adolescent marijuana use: differences by race/ ethnicity and sex. Drug Alcohol Depend. 2015;155:8-15 https://doi.org/10. 1016/j.drugalcdep.2015.08.025.

Karila L, Roux P, Rolland B, Benyamina A, Reynaud M, Aubin H-J, Lançon C. Acute and long-term effects of cannabis use: a review. Curr Pharm Des. 2014;20: $4112-8$.

Karjalainen K, Savonen J, Hakkarainen P, Väestökyselyt H. Suomalaisten huumeiden käyttö ja huumeasenteet - Huumeaiheiset väestökyselyt Suomessa 1992-2014. THL - Raportti 2/2016; 2016.

Karriker-Jaffe KJ. Neighborhood socioeconomic status and substance use by U.S. adults. Drug Alcohol Depend. 2013;133(1):212-21 https://doi.org/10.1016/j. drugalcdep.2013.04.033.

Kedzior KK, Laeber LT. A positive association between anxiety disorders and cannabis use or cannabis use disorders in the general population- a metaanalysis of 31 studies. BMC Psychiatry. 2014;14:136 https://doi.org/10.1186/ 1471-244X-14-136.

Knaappila N, Marttunen M, Fröjd S, Lindberg N, Kaltiala-Heino R. Socioeconomic trends in school bullying among Finnish adolescents from 2000 to 2015. Child Abuse Negl. 2018;86:100-8 https://doi.org/10.1016/j.chiabu.2018.09.011.

Knaappila N, Marttunen M, Fröjd S, Lindberg N, Kaltiala-Heino R. Socioeconomic trends in adolescent smoking in Finland from 2000 to 2015. J Adolesc Health. 2019a; https://doi.org/10.1016/j.jadohealth.2018.11.017.

Knaappila N, Marttunen M, Fröjd S, Lindberg N, Kaltiala-Heino R. Changes in delinquency according to socioeconomic status among Finnish adolescents from 2000 to 2015b. Scand J Child Adolesc Psychiatry Psychol. 2019b;7:52-9 https://doi.org/10.21307/sjcapp-2019-008.

Koskinen S, Martelin T. Nykyiset kansanterveysongelmat ja mahdollisuudet niiden torjumiseen. Teoksessa: Terveydenhuollon menojen hillintä: rahoitusjärjestelmän ja ennaltaehkäisyn merkitys. Helsinki; 2007.

Krieger N, Williams DR, Moss NE. Measuring social class in US Public Health Research: concepts, methodologies, and guidelines. Annu Rev Public Health. 1997;18:341-78 https://doi.org/10.1146/annurev.publhealth.18.1.341.

Lund Jensen N, Pedersen HS, Vestergaard M, Mercer SW, Glümer C, Prior A. The impact of socioeconomic status and multimorbidity on mortality: a population-based cohort study. Clin Epidemiol. 2017;9:279-89 https://doi.org/ 10.2147/CLEP.S129415. 
Lund M. Social inequality in cigarette consumption, cigarette dependence, and intention to quit among Norwegian smokers. Biomed Res Int. 2015;2015: Article ID 835080 https://doi.org/10.1155/2015/835080.

MacDonald K, Pappas K. WHY NOT POT?: a review of the brain-based risks of cannabis. Innov Clin Neurosci. 2016;13:13-22.

Matthiessen J, Stockmarr A, Biltoft-Jensen A, Fagt S, Zhang H, Groth MV. Trends in overweight and obesity in Danish children and adolescents: 2000-2008 exploring changes according to parental education. Scand J Public Health. 2014;42:385-92 https://doi.org/10.1177/1403494813520356

Melchior M, Choquet M, Le Strat Y, Hassler C, Gorwood P. Parental alcohol dependence, socioeconomic disadvantage and alcohol and cannabis dependence among young adults in the community. Eur Psychiatry. 2011;26: 13-7 https://doi.org/10.1016/j.eurpsy.2009.12.011.

Moraeus L, Lissner L, Sjöberg A. Stable prevalence of obesity in Swedish schoolchildren from 2008 to 2013 but widening socio-economic gap in girls. Acta Paediatr. 2014;103:1277-84 https://doi.org/10.1111/apa.12785.

Nurmi J, Kaskela T, Perälä J, Oksanen A. Seller's reputation and capacity on the illicit drug markets: 11-month study on the Finnish version of the Silk Road. Drug Alcohol Depend. 2017;178:201-7 https://doi.org/10.1016/j.drugalcdep. 2017.05.018.

$\varnothing$ vrum A. Socioeconomic status and lifestyle choices: evidence from latent class analysis. Health Econ. 2011;20:971-84 https://doi.org/10.1002/hec.1662.

Patel S, Williams JW Jr, Wallace RB. What we do (and don't) know about the health effects of cannabis and whether marijuana is medicine. Ann Intern Med. 2017;166:747 https://doi.org/10.7326/M17-0501.

Pedersen W, Mastekaasa A, Wichstrøm L. Conduct problems and early cannabis initiation: a longitudinal study of gender differences. Addiction. 2001;96:41531 https://doi.org/10.1080/0965214002005392.

Rawshani A, Svensson A-M, Zethelius B, Eliasson B, Rosengren A, Gudbjörnsdottir S. Association between socioeconomic status and mortality, cardiovascular disease, and cancer in patients with type 2 diabetes. JAMA Intern Med. 2016; 176:1146 https://doi.org/10.1001/jamainternmed.2016.2940.

Robinson-Cimpian JP. Inaccurate estimation of disparities due to mischievous responders. Educ Res. 2014;43:171-85 https://doi.org/10.3102/ 0013189X14534297.

Rotko T, Aho T, Mustonen N, Linnanmäki E. Kapeneeko kuilu? Tilannekatsaus terveyserojen kaventamiseen Suomessa 2007-2010. Terveyden ja hyvinvoinnin laitos (THL). Raportti 8/2011; 2011.

Saydah SH, Imperatore G, Beckles GL. Socioeconomic status and mortality: contribution of health care access and psychological distress among U.S. adults with diagnosed diabetes. Diabetes Care. 2013;36:49-55 https://doi.org/ 10.2337/dc11-1864

Sleskova M, Salonna F, Geckova AM, Nagyova I, Stewart RE, van Dijk JP, Groothoff JW. Does parental unemployment affect adolescents' health? J Adolesc Health. 2006;38(5):527-35 https://doi.org/10.1016/j.jadohealth.2005.03.021.

Stringhini S, Dugravot A, Shipley M, Goldberg M, Zins M, Kivimäki M, Marmot M, Sabia S, Singh-Manoux A. Health behaviours, socioeconomic status, and mortality: further analyses of the British Whitehall II and the French GAZEL prospective cohorts. PLoS Med. 2011;8(2):e1000419 https://doi.org/10.1371/ journal.pmed.1000419.

Swift W, Coffey C, Carlin JB, Degenhardt L, Patton GC. Adolescent cannabis users at 24 years: trajectories to regular weekly use and dependence in young adulthood. Addiction. 2008;103:1361-70 https://doi.org/10.1111/j.1360-0443. 2008.02246.x

ter Bogt TFM, de Looze M, Molcho M, Godeau E, Hublet A, Kokkevi A, Kuntsche E, Nic Gabhainn S, Franelic IP, Simons-Morton B, Sznitman S, Vieno A, Vollebergh W, Pickett W. Do societal wealth, family affluence and gender account for trends in adolescent cannabis use? A 30 country cross-national study. Addiction. 2014;109:273-83 https://doi.org/10.1111/add.12373.

Thompson KM. Marijuana use among adolescents: trends, patterns, and influences. Minerva Pediatr. 2001;53:313-23.

Torikka A, Kaltiala-Heino R, Luukkaala T, Rimpelä A. Trends in alcohol use among adolescents from 2000 to 2011: the role of socioeconomic status and depression. Alcohol Alcohol. 2017;52:95-103 https://doi.org/10.1093/alcalc/agw048.

Torikka A, Kaltiala-Heino R, Rimpelä A, Marttunen M, Luukkaala T, Rimpelä M. Selfreported depression is increasing among socio-economically disadvantaged adolescents - repeated cross-sectional surveys from Finland from 2000 to 2011. BMC Public Health. 2014;14:408 https://doi.org/10.1186/1471-2458-14-408.

Tu AW, Ratner PA, Johnson JL. Gender differences in the correlates of adolescents' cannabis use. Subst Use Misuse. 2008:43:1438-63 https://doi. org/10.1080/10826080802238140.
Varjonen V. Finland drug situation 2012: 2012 national report to the EMCDDA: New developments, trends and in-depth information on selected issues. Helsinki: Terveyden ja hyvinvoinnin laitos; 2012.

Volkow ND, Baler RD, Compton WM, Weiss SRB. Adverse health effects of marijuana use. N Engl J Med. 2014;370:2219-27 https://doi.org/10.1056/ NEJMra1402309.

von Sydow K, Lieb R, Pfister H, Höfler M, Wittchen $\mathrm{H}-\mathrm{U}$. What predicts incident use of cannabis and progression to abuse and dependence? A 4-year prospective examination of risk factors in a community sample of adolescents and young adults. Drug Alcohol Depend. 2002;68:49-64.

Wang N, Cao F, Liu F, Jia Y, Wang J, Bao C, Wang X, Song Q, Tan B, Cheng Y. The effect of socioeconomic status on health-care delay and treatment of esophageal cancer. J Transl Med. 2015;13:241 https://doi.org/10.1186/s12967015-0579-9.

Wardle J, Steptoe A. Socioeconomic differences in attitudes and beliefs about healthy lifestyles. J Epidemiol Community Health. 2003;57:440-3 https://doi. org/10.1136/JECH.57.6.440.

Weitoft GR, Rosén M, Ericsson Ö, Ljung R. Education and drug use in Sweden-a nationwide register-based study. Pharmacoepidemiol Drug Saf. 2008;17: 1020-8 https://doi.org/10.1002/pds.1635.

Widome R, Wall M, Laska M, Neumark-Sztainer D. Adolescence to young adulthood: when socio-economic disparities in substance use emerge. Subst Use Misuse. 2013:48:1522-9.

Willis PE. Learning to labour: how working class kids get working class jobs. Farnborough: Saxon House; 1977.

Wu L-T, Swartz MS, Brady KT, Hoyle RH. Perceived cannabis use norms and cannabis use among adolescents in the United States. J Psychiatr Res. 2015; 64:79-87 https://doi.org/10.1016/J.JPSYCHIRES.2015.02.022.

Zoccolillo M, Vitaro F, Tremblay RE. Problem drug and alcohol use in a community sample of adolescents. J Am Acad Child Adolesc Psychiatry. 1999;38:900-7 https://doi.org/10.1097/00004583-199907000-00021.

\section{Publisher's Note}

Springer Nature remains neutral with regard to jurisdictional claims in published maps and institutional affiliations.

\section{Ready to submit your research? Choose BMC and benefit from:}

- fast, convenient online submission

- thorough peer review by experienced researchers in your field

- rapid publication on acceptance

- support for research data, including large and complex data types

- gold Open Access which fosters wider collaboration and increased citations

- maximum visibility for your research: over 100M website views per year

At $\mathrm{BMC}$, research is always in progress.

Learn more biomedcentral.com/submission 